\title{
Patient Descriptions of Rectal Effluents May Help to Predict the Quality of Bowel Preparation With Photographic Examples
}

\author{
Hoonsub So ${ }^{1}$, Sun-Jin Boo ${ }^{2}$, Hyungil Seo ${ }^{1}$, Ho-Su Lee ${ }^{1}$, Hyojeong Lee ${ }^{1}$, Sang Hyoung Park ${ }^{1}$, Kyung-Jo Kim ${ }^{1}$, \\ Byong Duk Ye ${ }^{1}$, Jeong-Sik Byeon ${ }^{1}$, Seung-Jae Myung ${ }^{1}$, Suk-Kyun Yang ${ }^{1}$, Jin-Ho Kim ${ }^{1}$, Seungbong Han ${ }^{3}$, \\ Dong-Hoon Yang ${ }^{1}$ \\ Departments of Gastroenterology, Asan Medical Center, University of Ulsan College of Medicine', Seoul, Departments of Internal Medicine, Jeju \\ National University School of Medicine ${ }^{2}$, Jeju, Department of Applied Statistics, Gachon University ${ }^{3}$, Seongnam, Korea
}

Background/Aims: Previous studies have suggested a weak correlation between self-reported rectal effluent status and bowel preparation quality. We aim to evaluate whether photographic examples of rectal effluents could improve the correlation between patient descriptions of rectal effluents and bowel preparation quality. Methods: Before colonoscopy, patients were asked to describe the nature of their last three rectal effluents. Photographic examples of rectal effluents were provided as a reference for scoring. Bowel preparation was subsequently assessed by a single endoscopist using a global preparation assessment scale. Preparation outcomes were grouped into two levels (excellent to good vs. fair to inadequate). Both univariate and multivariate logistic regression models were used to find any association between bowel preparation quality and patient characteristics. Results: A total of 138 patients completed the questionnaires. The mean age was $56.5 \pm 10.4$ years. The mean sum of the last three rectal effluent scores was 5.9 2.0. Higher rectal effluent scores (odds ratio [OR], 0.82; $P=0.043$ ) and the presence of diverticula $(\mathrm{OR}, 0.16 ; P<0.001)$ were risk factors for suboptimal preparation. Conclusions: Photographic example-guided patient descriptions of rectal effluents showed a statistically significant association with bowel preparation quality. However, clinical significance seemed to be low. The presence of diverticula was an independent predictive factor for suboptimal bowel preparation quality. (Intest Res 2015;13:153-159)

Key Words: Rectal effluent; Bowel preparation; Colonoscopy

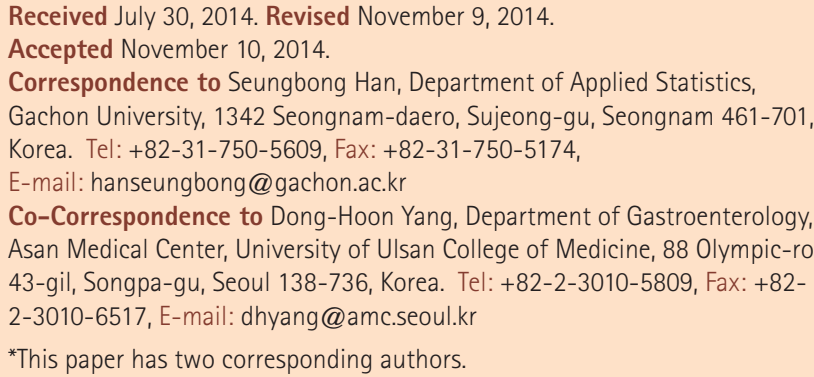

Financial support: None. Conflict of interest: None.

\section{INTRODUCTION}

Colonoscopy is now widely accepted as the standard method for the early detection of colon cancer. However, the miss rate for colon neoplasms, especially small-sized polyps, makes this method imperfect, and may lead to failure to prevent colon cancer. ${ }^{1}$ Poor bowel preparation is one of the factors associated with the an increased miss rate for colon polyps. ${ }^{2}$ Poor bowel preparation not only increases the risk of missing lesions, but also leads to canceled procedures, prolonged procedural times, and increased costs and rates of complcations. ${ }^{3-5}$ Hence, bowel preparation adequacy is gaining importance in both screening and surveillance

\footnotetext{
(c) Copyright 2015. Korean Association for the Study of Intestinal Diseases. All rights reserved.

This is an Open Access article distributed under the terms of the Creative Commons Attribution Non-Commercial License (http://creativecommons.org/licenses/by-nc/3.0)

which permits unrestricted non-commercial use, distribution, and reproduction in any medium, provided the original work is properly cited.
} 
tests. Some predictors, such as inactive lifestyles, diabetes, and cirrhosis have been suggested to be associated with poor bowel preparation. A method is required for predicting bowel preparation quality before initiating procedures. Patients are often asked about the appearance of their rectal effluents as a means of predicting bowel preparation before colonoscopy, but previous studies have suggested that patient self-assessment of bowel preparation is not reliable and is weakly correlated with bowel preparation statuses assessed by endoscopists. ${ }^{6,7}$ However, in these earlier studies, the questions related to rectal effluents were not objective. Accordingly, we aimed to evaluate whether photographic examples of rectal effluents could improve correlations between patient descriptions rectal effluents and actual bowel preparation quality.

\section{METHODS}

\section{Selection of Patients}

Patients who underwent a colonoscopy at Asan Medical Center, Seoul, Korea, were enrolled in this prospective and observational study between September 2010 and November 2010. During this period, 432 patients underwent a colonoscopy performed by a single endoscopist (Yang DH). $4 \mathrm{~L}$ of polyethylene glycol (PEG) solution was prescribed for bowel preparation. The following patients were not asked to participate in this survey: patients who were illiterate, who underwent emergency colonoscopy, or who had a history of bowel resection. A final series of 138 patients completed our questionnaire. The study was approved by the institutional review board of Asan Medical Center (Approval Number: S2014-1254-0001).
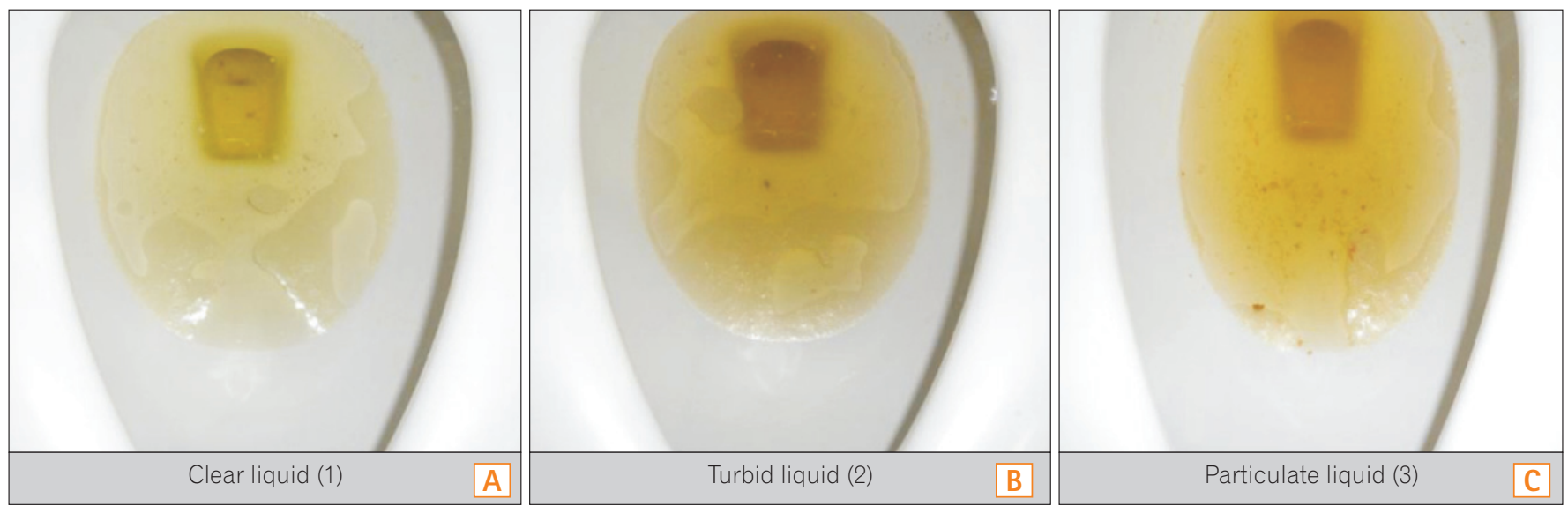

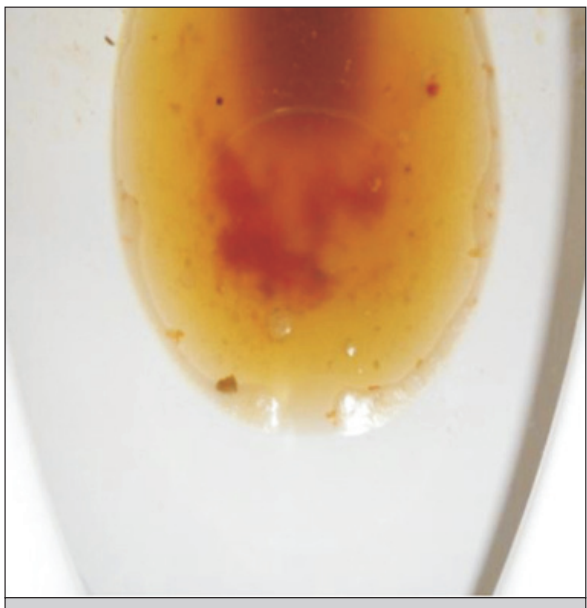

Liquid with small amount of feces (4)

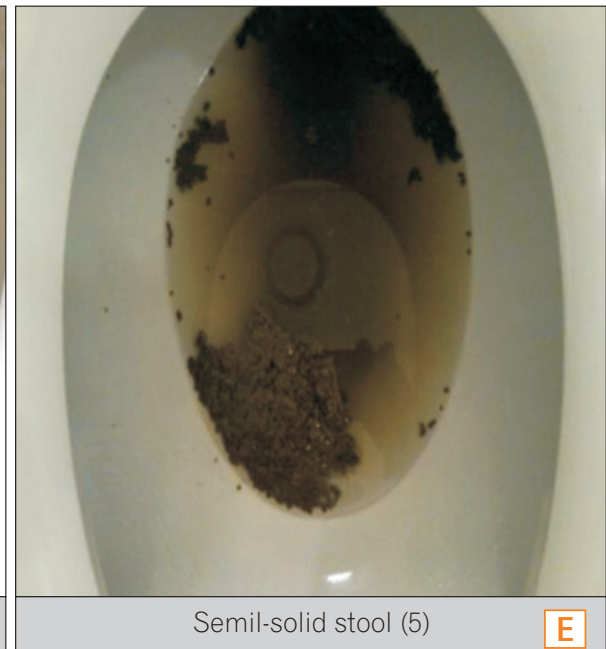

Fig. 1. Photographic examples of rectal effluent shown to patients for reference. (A) Clear liquid (score 1); (B) turbid liquid (score 2); (C) particulate liquid (score 3); (D) liquid with small amount of feces (score 4); (E) semi-solid stool (score 5). 


\section{Method}

Patients were asked to fill out a questionnaire that included patient factors (age, sex, body weight, height, history of abdominal surgery, objectives of the examination, and defecation frequency), procedural factors (amount of bowel preparation agent taken, time interval between the last PEG dose and colonoscopy), and the nature of the patient's last three consecutive rectal effluents. Five photographic examples of rectal effluents were provided as a reference and scored as follows: 1 , clear liquid; 2 , turbid liquid; 3 , particulate liquid; 4, liquid with small amounts of feces; and 5, semi-solid stool (Fig. 1). Therefore, if the sum of the last three consecutive rectal effluent score was 3 , this represented the clearest rectal effluent status possible. The result of the questionnaire was blinded to the endoscopist. The quality of bowel preparation was assessed by using Aronchick scale i.e., excellent, no more than small bits of adherent feces/fluid; good, small amounts of feces or fluid not interfering with the exam; fair, enough feces or fluid to prevent a completely reliable exam: poor, large amount of fecal residue requiring additional cleansing; inadequate, re-preparation needed. ${ }^{8}$ These five scales of bowel preparation were divided into two categories: the optimal group included the patients ranging from excellent to good preparation, and the suboptimal group from fair to inadequate preparation. The endoscopist routinely described the presence of diverticula. All other descriptions related to the colonoscopic findings were per-

Table 1. Characteristics of Enrolled Patients

\begin{tabular}{|c|c|c|c|c|}
\hline Variables & All patients $(n=138)$ & Suboptimal group $(n=44)$ & Optimal group $(n=94)$ & $P$-value \\
\hline Age (yr) & $56.5 \pm 10.4$ & $56.1 \pm 13.2$ & $56.7 \pm 8.9$ & 0.787 \\
\hline Male gender & $81(58.7)$ & $27(61.4)$ & $54(57.4)$ & 0.713 \\
\hline $\mathrm{BMI}\left(\mathrm{kg} / \mathrm{m}^{2}\right)$ & $24.0 \pm 3.4$ & $24.5 \pm 3.7$ & $23.7 \pm 3.2$ & 0.196 \\
\hline Previous intra-abdominal surgery & $36(26.3)$ & $12(27.9)$ & $24(25.5)$ & 0.835 \\
\hline Hypertension & $42(30.4)$ & $18(40.9)$ & $24(25.5)$ & 0.077 \\
\hline Diabetes mellitus & $17(12.3)$ & $6(13.6)$ & $11(11.7)$ & 0.784 \\
\hline Hypothyroidism & $4(2.9)$ & $3(6.8)$ & $1(1.1)$ & 0.095 \\
\hline \multicolumn{5}{|l|}{ Frequency of defecation } \\
\hline$\geq 1 /$ day & 115 (83.3) & 39 (88.6) & $76(80.9)$ & 0.330 \\
\hline$<2-3 /$ wk & $23(16.7)$ & $5(11.4)$ & $18(19.1)$ & \\
\hline \multicolumn{5}{|l|}{ Time of examination } \\
\hline Morning session & $65(47.1)$ & 19 (43.2) & $46(48.9)$ & 0.585 \\
\hline Afternoon session & $73(52.9)$ & $25(56.8)$ & $48(51.1)$ & \\
\hline \multicolumn{5}{|l|}{ Interval between preparation and exam } \\
\hline$<4$ or $6-12 h$ & $52(37.7)$ & $12(27.3)$ & $40(42.6)$ & 0.093 \\
\hline $4-6 h$ & $86(62.3)$ & $32(72.7)$ & $54(57.4)$ & \\
\hline PEG amount taken (mL) & $3,978 \pm 241$ & $3,966 \pm 294$ & $3,984 \pm 213$ & 0.927 \\
\hline \multicolumn{5}{|l|}{ Indication of colonoscopy } \\
\hline Polypectomy & $67(48.6)$ & $20(45.5)$ & $47(50)$ & 0.319 \\
\hline Screening & $29(21)$ & $9(20.5)$ & $20(21.3)$ & \\
\hline Past history of polypectomy & $20(14.5)$ & $6(13.6)$ & $14(14.9)$ & \\
\hline Diagnostic evaluation of gastrointestinal symptoms & $17(12.3)$ & $5(11.4)$ & $12(12.8)$ & \\
\hline Fever of unknown origin & $1(0.7)$ & $1(2.3)$ & $0(0)$ & \\
\hline $\mathrm{IBD}$ & $4(2.9)$ & $3(6.8)$ & $1(1.1)$ & \\
\hline Sum of the last three rectal effluents scores & $5.88 \pm 2.03$ & $6.43 \pm 2.03$ & $5.63 \pm 1.98$ & 0.034 \\
\hline
\end{tabular}

Values are presented as $n(\%)$ or mean \pm SD.

PEG, polyethylene glycol. 
Table 2. The Last Rectal Effluent Score and Bowel Preparation Quality Results

\begin{tabular}{lccccc}
\hline \multirow{2}{*}{$\begin{array}{c}\text { Bowel preparation } \\
\text { quality }\end{array}$} & $\begin{array}{c}\text { Clear liquid, } \\
\text { score } \mathbf{1}(\mathbf{n}=\mathbf{8 0})\end{array}$ & $\begin{array}{c}\text { Turbid liquid, } \\
\text { score } \mathbf{2}(\mathbf{n}=\mathbf{5 1})\end{array}$ & $\begin{array}{c}\text { Particulate liquid, } \\
\text { score } \mathbf{3}(\mathbf{n}=\mathbf{6})\end{array}$ & $\begin{array}{c}\text { Liquid with small amount } \\
\text { of feces, score } \mathbf{4}(\mathbf{n}=\mathbf{1})\end{array}$ & $\begin{array}{c}\text { Semi-solid stool, } \\
\text { score } \mathbf{5}(\mathbf{n}=\mathbf{0})\end{array}$ \\
\hline Excellent & $5(6.2)$ & $1(2.0)$ & 0 & 0 & 0 \\
Good & $53(66.3)$ & $32(62.7)$ & $3(50.0)$ & 0 & 0 \\
Fair & $19(23.8)$ & $15(29.4)$ & $1(16.7)$ & 0 & 0 \\
Poor & $3(3.7)$ & $3(5.9)$ & $2(33.3)$ & $1(100)$ & 0 \\
Inadequate & 0 & 0 & 0 & 0 & 0 \\
\hline
\end{tabular}

Values are presented as $n(\%)$.

formed by the endoscopist immediately after the procedure.

\section{Statistical Analysis}

Continuous variables were compared using Student's $t$ test or the Wilcoxon rank-sum test, and categorical variables were compared using the chi-square test or Fisher's exact test, as appropriate. Preparation outcomes were grouped into two levels (excellent to good $v$ s. fair to inadequate). Both univariate and multivariate logistic regression models were applied to identify any association between bowel preparation quality and patient characteristics. Receiver operating characteristic (ROC) analysis was conducted to identify the cut-off level for suboptimal preparation. Analyses were performed using R software, version 2.15.2 (R Foundation for Statistical Computing, Vienna, Austria) and SPSS 12.0 for Windows (SPSS Inc., Chicago, IL, USA). All reported $P$ values are two-sided, and $P$-values of less than 0.05 were considered as statistically significant.

\section{RESULTS}

\section{Patient Characteristics and Demographic Features}

A total of 138 patients participated in this study and completed the study questionnaire. The mean patient age was 56.5 years ( $\mathrm{SD}=10.4)$ and 81 of the $138(58.7 \%)$ patients were male. The mean BMI was $24.0 \mathrm{~kg} / \mathrm{m}^{2}$ ( $\mathrm{SD}=3.4$ ). The indications of colonoscopy included removal of colon polyps detected in other clinics ( $\mathrm{n}=67)$, screening for colorectal cancer $(\mathrm{n}=29)$, surveillance after previous polypectomy $(\mathrm{n}=20)$, follow up of IBD ( $\mathrm{n}=4)$, work-up for fever of unknown origin, and other assorted abdominal symptoms $(\mathrm{n}=17)$. Forty patients (29.0\%) had a history of previous abdominal surgery. Colonoscopy was performed in the morning session for 65 patients (47.1\%) and in the afternoon session for the remain-

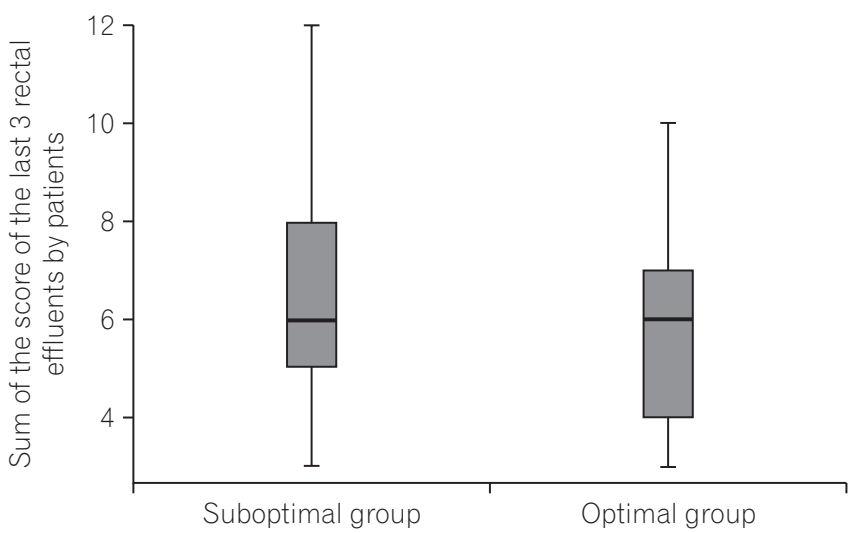

Fig. 2. Box plot of the sum of the scores of the three rectal effluents between the two study groups. The box plot shows the median and range of each group. The suboptimal and optimal groups showed the same median value of 6 , with ranges of $3-12$ and $3-10$, respectively.

ing 73 patients. The mean amount of PEG taken was 3,978 $\mathrm{mL}(\mathrm{SD}=241)$. The mean value of the last three rectal effluents was 5.88 ( $\mathrm{SD}=2.03)$. Baseline characteristics and demographic features are summarized in Table 1.

\section{Results of Colonoscopy}

Cecal intubation was performed in all procedures. The endoscopist rated the quality of bowel preparation of his/her patients as follows: 6 , excellent; 88 , good; 35 , fair; and 9, poor. Normal findings were seen in 41 patients. Eighty-seven patients had non-advanced adenomas, and 23 had advanced adenomas. Three patients had early colorectal cancer and one patient had advanced colorectal cancer. Four patients had IBDs and one had colonic lymphoma. None of the patients had obstructive lesions.

Finally, 25 patients had one or more colonic diverticula and 24 of these had diverticula in their cecum or ascending colon. Last rectal effluent scores and the result of bowel 
preparation quality are summarized in Table 2.

\section{Factors Associated With Suboptimal Preparation}

Patients were divided into two groups according to their bowel preparation status: optimal (excellent to good) or suboptimal (fair to inadequate). The mean last rectal effluent score was not different between the two groups $(1.41 \pm 0.56$ in the optimal group and $1.61 \pm 0.72$ in the suboptimal group, $P=0.111)$. The mean of the last three consecutive rectal effluent scores was higher in the suboptimal group than in the optimal group ( $6.43 \pm 2.03$ vs. $5.63 \pm 1.98, P=0.034)$. However, the median values of the sum of the last three consecutive rectal effluent scores were not different between the two groups (6 [range 3-12] vs. 6 [range 3-10]) (Fig. 2). According to univariate regression analysis, the presence of colonic diverticula and the sum of the last three rectal effluent scores showed significant associations with suboptimal bowel preparation quality (Table 3). In multivariate regression analysis, the presence of diverticula (OR, 0.16; 95\% CI, 0.06-0.43; $P<0.001)$ and the sum of the last three rectal effluent scores (OR, 0.82; 95\% CI, 0.68-0.99; $P=0.043$ ) remained significant (Table 4). ROC analysis was performed to identify the value of the last three consecutive rectal effluent scores that could differentiate suboptimal bowel preparation status, and 4 was the best cut-off value, with 0.955 sensitivity, 0.202 specificity, 0.359 positive predictive value, 0.905 negative predictive value, and 0.608 (95\% CI, 0.510-0.706) area under the curve (Fig. 3).

Table 3. Univariate Regression Analysis of Variables Associated With Optimal Bowel Preparation

\begin{tabular}{|c|c|c|}
\hline Variables & OR $(95 \% \mathrm{Cl})$ & $P$-value \\
\hline Age & $1.00(0.97-1.04)$ & 0.778 \\
\hline Female gender & $1.18(0.57-2.45)$ & 0.663 \\
\hline $\mathrm{BMI}$ & $0.93(0.84-1.04)$ & 0.196 \\
\hline Previous intra-abdominal surgery & 0.89 (0.39-1.99) & 0.770 \\
\hline Hypertension & $0.50(0.23-1.06)$ & 0.070 \\
\hline Diabetes mellitus & $0.84(0.29-2.44)$ & 0.748 \\
\hline Hypothyroidism & $0.15(0.01-1.46)$ & 0.101 \\
\hline Constipation & $0.62(0.24-1.59)$ & 0.325 \\
\hline Frequency of defecation & $1.85(0.64-5.35)$ & 0.258 \\
\hline \multicolumn{3}{|l|}{ Time of examination } \\
\hline Morning session & Reference & \\
\hline Afternoon session & $0.79(0.39-1.63)$ & 0.528 \\
\hline \multicolumn{3}{|l|}{ Interval between preparation \& colonoscopy } \\
\hline$<4$ or $6-12 \mathrm{~h}$ & Reference & \\
\hline $4-6 h$ & $0.51(0.23-1.10)$ & 0.090 \\
\hline PEG amount taken ( $\geq 90 \%)$ & $0.77(0.18-3.37)$ & 0.726 \\
\hline Sum of the score of the last 3 rectal effluents (per 1 score increment) & $0.82(0.69-0.98)$ & 0.032 \\
\hline Presence of diverticulum & $0.19(0.07-0.47)$ & $<0.001$ \\
\hline
\end{tabular}

PEG, polyethylene glycol.

Table 4. Multivariable Regression Analysis of Variables Associated With Optimal Bowel Preparation

\begin{tabular}{lcr}
\multicolumn{1}{c}{ Variables } & OR (95\% Cl) & \multicolumn{1}{c}{$\boldsymbol{P}$-value } \\
\hline Sum of the scores of the last three rectal effluents (per 1 score increment) & $0.82(0.68-0.99)$ & 0.043 \\
Presence of diverticulum & $0.16(0.06-0.43)$ & $<0.001$ \\
\hline
\end{tabular}




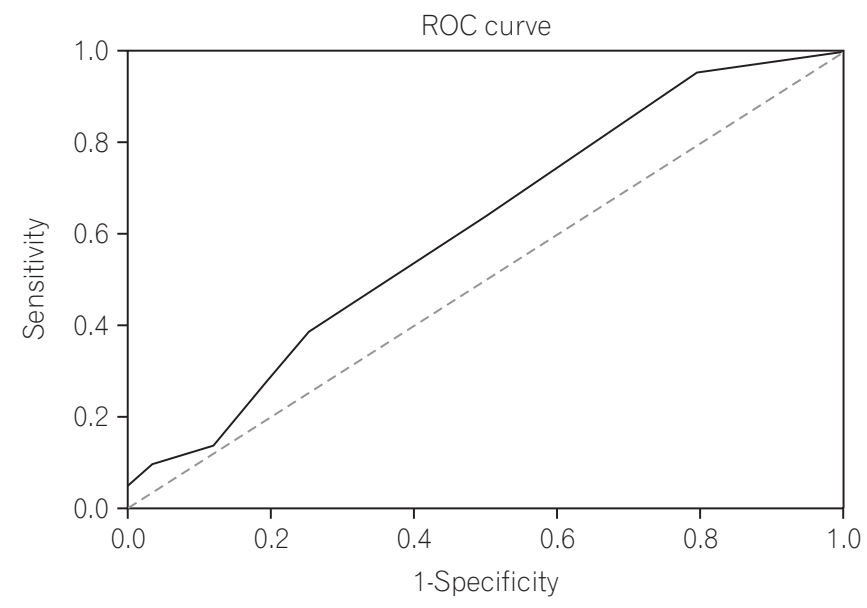

Fig. 3. Receiver-operating-characteristic (ROC) curve for discriminating suboptimal bowel preparation status with three consecutive rectal effluent scores. The sensitivity, specificity, area under the curve, and cutoff point for suboptimal bowel preparation were $0.955,0.202,0.608$, and 4 , respectively.

\section{DISCUSSION}

Previous studies have suggested that patient assessments of bowel preparation are not reliable. ${ }^{6,7}$ Harewood et al. ${ }^{7}$ reported that patient assessments of their own bowel preparation quality, based on the nature of the rectal effluent, are not reliable as patients tended to overestimate the cleanliness of their colon. Fatima et al. ${ }^{6}$ reported only a slight agreement between the patient's description of their effluent and the colonoscopist's description of the bowel preparation. However, in these studies, the questions relating to the status of the rectal effluent after administration of the bowel preparation regimen were somewhat subjective and lacked clarity. We hypothesized that guidance provided by photographic examples of rectal effluent might help to improve the correlation between self-assessments of rectal effluents and bowel preparation quality assessments made by endoscopists. Moreover, we also asked patients to rate the status of their last three consecutive rectal effluents because merely describing the status of the latest rectal effluent might not be sufficient to estimate the true bowel preparation status. Indeed, the sum of the last three rectal effluent scores was found to be associated with bowel preparation status in our study. However, despite showing statistical significance, the OR was relatively small, and the median values of the sums of the last three consecutive rectal effluent scores in the two groups were the same. According to ROC analysis, the cutoff level was 4 for differentiating the suboptimal group from the optimal group. However, specificity and area under the curve value were insufficient to confirm this cut-off value. To determine the precise cut-off level, further investigation is needed with more patients. Hence, photographic rectal effluents scores based on photographic example guidance could not guarantee sufficient bowel preparation status.

Many risk factors for poor bowel preparation have previously been demonstrated. For example, later colonoscopy starting time, failure to follow preparation instructions, inpatient status, procedural indication of constipation, history of taking tricyclic antidepressants, male sex, history of stroke or dementia, history of cirrhosis, lower patient activation, and obesity have been found to be associated with poor bowel preparation status. ${ }^{9-11}$ In the present study, we also tried to identify other predictive factors for bowel preparation status. Unprecedentedly, this study showed that the presence of colonic diverticulosis was a significant risk factor for suboptimal bowel preparation. To our knowledge, the association between bowel preparation and colonic diverticulosis has not been intensively investigated. Most diverticula in Asian patients are located in the right side of the colon. ${ }^{12,13}$ As the right side of the colon is larger, feces filling the hollow space may not be cleared by the standard amount of agent administered. Recent studies into interval colorectal cancer have suggested that interval colorectal cancer was associated with the presence of diverticular diseases, which may itself hinder adequate observation because of the structural characteristics of colons with diverticulosis. ${ }^{14-16}$ However, based on the findings of the present study, diverticula also affect bowel preparation quality, and may also be related with interval colorectal cancer. Further studies should be performed to identify the association between diverticular diseases and the quality of bowel preparation, and reanalyzing the raw data collected from the several prospective bowel preparation studies in Korea could be the first step toward assessing the association between bowel preparation quality and di-

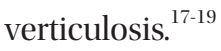

Our study has several limitations. First, this study was performed by a single endoscopist in a single hospital, and selection bias might have influenced the results, although the endoscopist was blind to the patients' responses to the questionnaire. Second, the number of patients was relatively small. Third, the endoscopist used the Aronchick scale to assess bowel preparation status. Although this is a clinically useful bowel preparation scale, it lacks the complexity that would be needed to accurately reflect bowel preparation quality. In conclusion, rectal effluent scores based on photographic examples showed statistically significant association with bowel preparation quality. However, according to 
ROC analysis, patients' descriptions of their rectal effluents could not effectively predict the quality of bowel preparation. The presence of diverticula is independently predictive of suboptimal bowel preparation, and further investigations are needed to confirm the association between the colonic diverticulosis and bowel preparation quality.

\section{REFERENCES}

1. van Rijn JC, Reitsma JB, Stoker J, Bossuyt PM, van Deventer SJ, Dekker E. Polyp miss rate determined by tandem colonoscopy: a systematic review. Am J Gastroenterol 2006;101:343-350.

2. Harewood GC, Sharma VK, de Garmo P. Impact of colonoscopy preparation quality on detection of suspected colonic neoplasia. Gastrointest Endosc 2003;58:76-79.

3. Froehlich F, Wietlisbach V, Gonvers JJ, Burnand B, Vader JP. Impact of colonic cleansing on quality and diagnostic yield of colonoscopy: the European Panel of Appropriateness of Gastrointestinal Endoscopy European multicenter study. Gastrointest Endosc 2005;61:378-384.

4. Lebwohl B, Kastrinos F, Glick M, Rosenbaum AJ, Wang T, Neugut AI. The impact of suboptimal bowel preparation on adenoma miss rates and the factors associated with early repeat colonoscopy. Gastrointest Endosc 2011;73:1207-1214.

5. Rex DK, Imperiale TF, Latinovich DR, Bratcher LL. Impact of bowel preparation on efficiency and cost of colonoscopy. Am J Gastroenterol 2002;97:1696-1700.

6. Fatima H, Johnson CS, Rex DK. Patients' description of rectal effluent and quality of bowel preparation at colonoscopy. Gastrointest Endosc 2010;71:1244-1252.

7. Harewood GC, Wright CA, Baron TH. Assessment of patients' perceptions of bowel preparation quality at colonoscopy. Am J Gastroenterol 2004;99:839-843.

8. Aronchick CA, Lipshutz WH, Wright SH, Dufrayne F, Bergman G. A novel tableted purgative for colonoscopic preparation: efficacy and safety comparisons with Colyte and Fleet PhosphoSoda. Gastrointest Endosc 2000;52:346-352.

9. Ness RM, Manam R, Hoen H, Chalasani N. Predictors of inadequate bowel preparation for colonoscopy. Am J Gastroenterol 2001;96:1797-1802.
10. Serper M, Gawron AJ, Smith SG, et al. Patient factors that affect quality of colonoscopy preparation. Clin Gastroenterol Hepatol 2014;12:451-457.

11. Borg BB, Gupta NK, Zuckerman GR, Banerjee B, Gyawali CP. Impact of obesity on bowel preparation for colonoscopy. Clin Gastroenterol Hepatol 2009;7:670-675.

12. Sugihara K, Muto T, Morioka Y, Asano A, Yamamoto T. Diverticular disease of the colon in Japan. A review of 615 cases. Dis Colon Rectum 1984;27:531-537.

13. Kim SY, Oh TH, Seo JY, et al. The clinical factors for predicting severe diverticulitis in Korea: a comparison with Western countries. Gut Liver 2012;6:78-85.

14. Cooper GS, Xu F, Schluchter MD, Koroukian SM, Barnholtz Sloan JS. Diverticulosis and the risk of interval colorectal cancer. Dig Dis Sci 2014;59:2765-2772.

15. Bressler B, Paszat LF, Chen Z, Rothwell DM, Vinden C, Rabeneck L. Rates of new or missed colorectal cancers after colonoscopy and their risk factors: a population-based analysis. Gastroenterology 2007;132:96-102.

16. Erichsen R, Baron JA, Stoffel EM, Laurberg S, Sandler RS, Sorensen HT. Characteristics and survival of interval and sporadic colorectal cancer patients: a nationwide population-based cohort study. Am J Gastroenterol 2013;108:1332-1340.

17. Kang MS, Kim TO, Seo EH, et al. Comparison of the efficacy and tolerability between same-day picosulfate and split-dose polyethylene glycol bowel preparation for afternoon colonoscopy: a prospective, randomized, investigator-blinded trial. Intest Res 2014;12:53-59.

18. Moon CM, Park DI, Choe YG, et al. Randomized trial of 2-L polyethylene glycol + ascorbic acid versus 4-L polyethylene glycol as bowel cleansing for colonoscopy in an optimal setting. J Gastroenterol Hepatol 2014;29:1223-1228.

19. Kim HG, Huh KC, Koo HS, et al. Sodium picosulfate with magnesium citrate (SPMC) plus laxative is a good alternative to conventional large volume polyethylene glycol in bowel preparation: a multicenter randomized single-blinded trial. Gut Liver doi: 10.5009/gnl14010. Published online ahead of print 7 October 2014 . 